\title{
Repensar el compromiso desde el quehacer etnográfico: incomodidades y potencialidades de la producción de conocimiento con organizaciones sociales
}

\author{
Rethinking Engagement from the Ethnographic \\ Experience: Discomforts and Potentialities of Knowledge \\ Production with Social Organizations
}

\author{
Florencia Trentini* \\ Conicet-Universidad Nacional de Quilmes \\ Sandra Wolanski** \\ Conicet-Universidad de Buenos Aires
}

\begin{abstract}
RESUMEN
Este artículo explora el quehacer etnográfico con organizaciones sociales, a partir de dos experiencias de investigación disímiles: con un sindicato urbano y con un proyecto de comanejo de un parque natural. Problematizamos la idea del compromiso como vínculo construido en nuestro trabajo y mostramos su carácter situado, moldeado en diálogo con los intereses y propuestas de las organizaciones, así como por las definiciones y sentidos locales del término.
\end{abstract}

Palabras clave: etnografía, compromiso, investigación activista, organizaciones sociales.

\begin{abstract}
$\overline{\text { ABSTRACT }}$
This paper explores ethnographic work with social organizations, drawing from two dissimilar research experiences: with an urban labor union and with a natural park co-management project. We problematize the notion of engagement as a specific relation built during our work and reveal its situated nature, shaped through dialogue with the interests and proposals of the organizations, as well as by local definitions and meanings.
\end{abstract}

Keywords: ethnography, engagement, activist research, social movements.

Doctora en Antropología de la Universidad de Buenos Aires. Investigadora en el Instituto de Estudios sobre Ciencia y Tecnología, Universidad Nacional de Quilmes. Becaria posdoctoral del Conicet. flortrentini@gmail.com.

** Doctora en Ciencias Antropológicas de la Universidad de Buenos Aires. Investigadora en el Instituto de Ciencias Antropológicas, Universidad de Buenos Aires. Becaria posdoctoral del Conicet. sandra.wolanski@gmail.com. 


\section{Introducción}

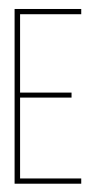

ste trabajo explora el quehacer etnográfico con organizaciones sociales, a partir del intercambio entre dos experiencias de investigación doctoral en contextos distintos y con temas, sujetos, problemáticas y preguntas disímiles. A pesar de las enormes diferencias, los vínculos y relaciones construidos en la investigación suscitaron una reflexión en común.

La primera investigación (Wolanski 2015) se centró en las prácticas del activismo de Foetra, el principal sindicato de las telecomunicaciones del Área Metropolitana de Buenos Aires, que reúne alrededor de 20.000 trabajadores de las empresas de telefonía fija y móvil que se desempeñan en las áreas de instalación, reparación y mantenimiento, así como de atención comercial, telefónica y administrativa. Es un sindicato de larga tradición, que se identifica y es reconocido por una historia combativa ${ }^{1}$ y por haber formado parte de importantes procesos de movilización en distintos periodos de la historia argentina, en particular, contra la privatización de la Empresa Nacional de Telecomunicaciones en 1990 (Wolanski, en prensa). La investigación doctoral se desarrolló en un periodo de grandes tensiones y contradicciones (2013-2017), pues el sindicato no solo no enfrentaba amenazas y conflictos con las empresas del sector ${ }^{2}$, sino que se encontraba en expansión, tanto en cantidad de afiliados, como en recursos. En efecto, durante los gobiernos de Néstor Kirchner y Cristina Fernández de Kirchner se produjo un fortalecimiento y hasta una revitalización de las organizaciones sindicales en Argentina (Etchemendy y Berins 2008; Senén y Haidar 2009). Esta coyuntura supuso un conjunto de reacomodamientos en la práctica sindical de Foetra y la redefinición de demandas, modos de movilización y relaciones dentro del sindicato. En este contexto, la investigación se centró en el trabajo militante de los activistas en la producción cotidiana de la organización sindical y, en particular, en los modos en que esa producción tenía lugar de manera disputada en las relaciones entre distintas generaciones de activistas. En el activismo se incluyen todos aquellos trabajadores vinculados de distintas maneras a la actividad gremial y que transitan cotidianamente los espacios del sindicato,

1 En este texto la bastardilla indica categorías de la práctica de los sujetos con los que trabajamos.

2 El sector de la telefonía fija en Argentina es un oligopolio compartido por dos empresas de operación internacional: Telefónica de Argentina S. A. y Telecom de Argentina. El sector de telefonía móvil está compuesto por cuatro grandes empresas: Movistar S. A. y Personal -vinculadas a las dos empresas ya mencionadas-, Nextel y Claro. 
cumplan o no funciones de responsabilidad gremial como delegados, secretarios o colaboradores.

La segunda investigación (Trentini 2014) analizó la implementación de la administración compartida o el comanejo de territorios y recursos entre el Parque Nacional Nahuel Huapi (PNNH) y las comunidades mapuche asentadas dentro de su jurisdicción. El caso presentaba una particularidad: la zona había sido históricamente considerada como "sin indios" debido a que, con la creación del parque en 1934, quienes habitaban la zona fueron categorizados genéricamente como privados (en su mayoría familias que habían ayudado a financiar la "conquista del desierto" y a cambio recibieron tierras y títulos de propiedad); otros (en su mayoría indígenas) clasificados como pobladores con Permisos Precarios de Ocupación y Pastaje (PPOP), a quienes se reconocen derechos de ocupación pero no de propiedad; e intrusos sin autorización para habitar dentro del parque, que debían ser desalojados. No hubo comunidades indígenas reconocidas oficialmente $^{3}$. Como resultado de la invisibilización y estigmatización de la identidad mapuche en una zona de frontera con Chile, en la región se había dado un fuerte proceso de desadscripción étnica (García y Valverde 2007; Méndez 2005; Radovich y Balazote 1999) ${ }^{4}$. A raíz de la creación del comanejo en el Parque Nacional Lanín en el año 2000, del reconocimiento de los derechos indígenas en Argentina en la década de los noventa y del avance de negocios turísticos e inmobiliarios sobre los territorios de pobladores precarios, el cambio en la política institucional de la Administración de Parques Nacionales generó un marco propicio para que algunas familias con PPOP comenzaran a revalorizar su historia, a reconocerse públicamente como mapuches y a conformarse de manera formal como comunidades indígenas, con personería jurídica otorgada por el Estado. La indigenización, sin embargo, ha sido fuertemente cuestionada por la sociedad local y el estado provincial como un invento oportunista para disputar territorios de importante valor económico.

La investigación de Trentini (2009-2017) tuvo lugar en un periodo de fuerte tensión entre las comunidades y la administración del parque, pues la readscripción étnica ponía en cuestión la autenticidad de los reclamos identitarios y territoriales, y en consecuencia, la posibilidad de incluir o no a los indígenas en

Ser catalogado como intruso se relacionaba con la importancia estratégica del territorio que estas familias ocupaban (para el desarrollo de emprendimientos turísticos, por ejemplo). Para conservar o renovar los PPOP se debía cumplir con una estricta normativa, pero la dificultad para acatarla fue convirtiendo a muchos pobladores en intrusos.

4 Los conflictos limítrofes entre Chile y Argentina y el nacionalismo oficial invisibilizaron y estigmatizaron la cuestión indígena al considerar que el reconocimiento de las poblaciones indígenas podría atentar contra la soberanía nacional. 
el proyecto de comanejo (Trentini 2016). La indagación se enfocó en el análisis de los procesos de construcción de la diferencia cultural y problematizó la articulación entre la identidad, la cultura y el territorio en comunidades mapuches consideradas "nuevas" - como las de Nahuel Huapi-en oposición a las comunidades "tradicionales” de las provincias de Río Negro y Neuquén, históricamente reconocidas por el Estado argentino.

En este artículo reflexionamos sobre las metodologías empleadas en nuestros trabajos, en particular, sobre los vínculos que establecimos y que en ambos casos definimos en términos de compromiso. El objetivo es problematizar dicha categoría desde nuestras prácticas etnográficas y mostrar que nuestro compromiso fue más allá del posicionamiento que adoptamos como antropólogas, en tanto fuimos interpeladas por nociones locales de compromiso y a la vez construimos nuestras propias modalidades comprometidas de manera relacional, en diálogo y negociación entre las orientaciones que asumimos como investigadoras y las definiciones y necesidades de los sujetos y organizaciones. Indagar en estas experiencias, en sus incomodidades y en sus potencialidades para la producción de conocimiento, tiene como objetivo contribuir a la reflexión sobre nuestra posición como antropólogas en los procesos de investigación y colaboración: cómo, por qué y para quién(es) producimos conocimientos.

El texto se organiza en tres apartados. En el primero, reconstruimos las investigaciones, los términos en que fuimos construyendo el compromiso y su carácter situado en experiencias y definiciones locales. En el segundo, interpelamos desde nuestras experiencias etnográficas algunas posturas metodológicas que proponen como punto de partida el compromiso ético o político del investigador con los sujetos, así como el potencial de la investigación social como herramienta para el cambio social, en sus múltiples definiciones. Finalmente, reflexionamos sobre los vínculos construidos - las tensiones que los atravesaron-y la producción de conocimiento.

\section{El compromiso desde el quehacer etnográfico}

Hace más de una década, iniciamos una fecunda relación con dos organizaciones sociales que partía de intereses y posicionamientos políticos e investigativos. Desde nuestras preocupaciones personales y desde el quehacer de los equipos en los que nos insertamos, buscábamos colaborar de algún modo con las causas de 
las organizaciones y los sujetos. Nuestros vínculos en campo mutaron a lo largo de ese proceso, según las relaciones (más o menos estables) que entablamos con nuestros interlocutores. Con el tiempo, hemos asumido diversas posiciones en campo y fuimos identificadas (y autoidentificadas) de distintas formas. Iniciamos nuestras investigaciones siendo estudiantes, pero cuando nos graduamos y nos convertimos en "verdaderas" antropólogas se produjo un cambio fundamental para nuestros interlocutores, en tanto nuestros títulos profesionales contribuyeron a validar nuestra práctica y participación en distintos proyectos. Continuamos nuestras tesis doctorales con esas mismas organizaciones, a la vez que desarrollamos otros proyectos de extensión/transferencia, investigación y construcción de instancias colectivas, como talleres o capacitaciones auspiciadas por las organizaciones. En este apartado reconstruimos los términos en que construimos la práctica de investigación y la centralidad de las propias organizaciones en su definición.

En el caso de Wolanski (2015)5 ${ }^{5}$, el trabajo de campo se llevó a cabo con los activistas de una de las agrupaciones que conforman la dirección del sindicato ${ }^{6}$, en dos ámbitos diferentes: la creación de un espacio para la juventud en el seno de la organización y la Secretaría de Cultura y Capacitación. El conocimiento previo de algunas activistas que habían sido protagonistas de la tesis de licenciatura y ahora eran parte de estos espacios facilitó el acceso inicial para la observación participante. Sin embargo, la vinculación con otro conjunto de actividades de la Secretaría de Cultura y Capacitación permitió conocer y ser testigo de las dinámicas cotidianas del activismo y discutir con sus protagonistas sobre los dilemas y tensiones de su práctica sindical7.

5 El Programa Procesos de Reconfiguración Estatal, Resistencia Social y Construcción de Hegemonías, del Instituto de Ciencias Antropológicas de la Universidad de Buenos Aires, proporcionó un marco de discusiones metodológicas en torno a la etnografía con organizaciones sociales (Fernández 2010, 2016; Fernández y Carenzo 2012). Metodológicamente, la tesis siguió un enfoque de antropología política sobre procesos de organización y modos de construcción colectivos.

$6 \quad$ El trabajo de campo se llevó a cabo con los activistas del Frente Telefónico - una de las cinco agrupaciones de Foetra-; allí se observaron las dinámicas desde dentro de la dirección y desde una agrupación posicionada en la izquierda de la institución. Por las dinámicas específicas del activismo de Foetra, esto supuso anular abiertamente la vinculación con algunas agrupaciones políticas y tomar todas las precauciones posibles en el manejo de la información sobre las dinámicas internas.

7 Entre el 2013 y el 2016 Wolanski fue colaboradora docente en el Programa de Fortalecimiento y Sistematización de la Práctica Sindical (Forsa), desarrollado por la Secretaría de Cultura y Capacitación de Foetra y el Ministerio de Trabajo, Empleo y Seguridad Social de la Nación. En ese marco participó en la planificación y coordinación de diez ediciones del curso que abarcaron a casi quinientos trabajadores de las telecomunicaciones, en la sistematización de los conocimientos producidos en formato de cartillas de los cursos, además de tres publicaciones sobre los contenidos generados. 
Tal inserción permitió examinar la práctica activista en claves que no eran evidentes en un principio, al exponer el trabajo pedagógico de formación de los activistas, en el que ciertos valores y normas implícitas, que distinguían las prácticas correctas de las incorrectas y las deseables de las indeseables, ocupaban un lugar central. A partir de esta observación, se analizaron la lucha, el compromiso y la participación - como categorías de la práctica activista- en tanto construcciones morales que permeaban las prácticas del activismo en Foetra y buscaban transmitirse a los y las jóvenes en particular (Wolanski 2015, en prensa). La participación también permitió observar el lugar de los jóvenes en el sindicato durante los últimos años y los modos en que las diferencias etarias y generacionales - más que de clase o género- se constituían en un lenguaje para procesar colectivamente procesos de cambio social y disputas políticas. Algunas de estas disputas afectaban la misma definición moral del compromiso y la participación de los activistas; los jóvenes eran acusados de ser militantes cómodos, acostumbrados a la militancia fácil, que no implicaba los sacrificios de otras épocas. Sin embargo, las prácticas de las que se los señalaba también se extendían al conjunto de los activistas. La investigación reveló que tales prácticas estaban relacionadas con la situación particular que atravesaba Foetra: por una parte, la ausencia de grandes conflictos con las empresas telefónicas y, por otra, el posicionamiento organizativo frente al gobierno de Cristina Fernández de Kirchner - considerado un aliado en un proyecto nacional común-, lo cual tensionaba y modificaba los modos de acción gremial en un sindicato que se asume como combativo y con una tradición de lucha.

Por su parte, la investigación de Trentini (2014) ${ }^{8}$ se centró en la construcción cotidiana del comanejo entre la administración del Parque Nacional Nahuel Huapi y las comunidades mapuche asentadas dentro de su jurisdicción. El trabajo de campo incluyó la observación participante, principalmente en las oficinas de comanejo en la Intendencia del PNNH, durante las visitas de técnicos de la institución a las comunidades, y en ceremonias, asambleas (trawvn) y reuniones zonales. El trabajo previo facilitó el acceso a estos espacios, no obstante, la confianza construida en otras actividades realizadas con aquellas abrió la puerta en distintas instancias institucionales y comunitarias donde fue testigo

8 La investigación se desarrolló en el marco del Proyecto UBACyT 2012-2015 "Etnicidades, movimientos y comunidades indígenas en contextos de promoción de emprendimientos productivos: una perspectiva comparativa entre los pueblos indígenas mapuche, chané y qom", FFyL-UBA, dirigido por el Dr. Sebastián Valverde. La tesis recupera diversos trabajos antropológicos que problematizaron la cuestión del compromiso, la antropología colaborativa y la investigación activista en relación con comunidades mapuche-tehuelche en Argentina (Cañumil y Ramos 2011; Kroppf 2014; Rodríguez 2010). 
de las discusiones, contradicciones, acuerdos y desacuerdos que implicaba la implementación cotidiana del comanejo. Estas actividades incluyeron diversos proyectos de extensión y transferencia que buscaban contar y visibilizar la otra historia de la región de Nahuel Huapi: la de las familias mapuche no reconocidas por el Estado argentino y que fueron categorizadas como pobladores o intrusos con la creación del parque.

El vínculo construido con las comunidades en el marco de estos proyectos -fundamentales para el fortalecimiento identitario y la reafirmación territorial-implicó la exposición a espacios, situaciones y personas que complejizaron el análisis del comanejo más allá de las definiciones institucionales y los posicionamientos públicos. A partir de esa inserción particular, la investigación doctoral quiso contribuir al estudio de la construcción de la diferencia cultural y problematizar la articulación entre la identidad, la cultura y el territorio que la propia categoría de comanejo movilizaba o prescribía, al definir la autenticidad e inautenticidad de los reclamos y las luchas de cada comunidad.

Durante la investigación hubo momentos de fuerte tensión entre las comunidades y organizaciones indígenas y la administración del parque, debido a que la institucionalidad construía una diferencia entre las "verdaderas" comunidades que representaban un éxito para el comanejo y aquellas que eran vistas como un problema, por no cumplir con los atributos prefigurados por la administración para definirlas como comunidades indígenas. Estas tensiones repercutieron en la investigación, ya que más de una vez fue necesario tomar posición en una disputa que ocurría al mismo tiempo que el trabajo de campo. Acompañar a la comunidad más problemática y conflictiva exigió una importante demostración del posicionamiento de la investigadora en el momento más álgido de la negociación entre las partes, actitud que fue entendida por las comunidades como una muestra del compromiso, y amplió las posibilidades de Trentini de etnografiar diversas instancias, vedadas para otros.

El análisis de la situación reveló diversas formas de ser comunidad (Trentini 2016) que requieren de una plasticidad institucional que no es posible garantizar con versiones prefiguradas y modelizadas de comanejo. Por otro lado, problematizó el concepto de comunidad indígena, al mostrar que en los marcos del comanejo estas no eran concebidas como productos históricos, sino como entidades homogéneas, que debían cumplir con ciertas condiciones preestablecidas para ser legitimadas e incorporadas al proyecto.

En los dos casos de investigación, compartir espacios de intervención (como docentes, talleristas, investigadoras, según los objetivos de la organización) nos situó, de manera similar, en un lugar de compañeras o aliadas que apoyaban y 
acompañaban los procesos organizativos y de fortalecimiento de los colectivos. El apelativo compañera(o), que opera como una categoría nativa, es central en el ámbito sindical y se retoma en los movimientos políticos y sociales argentinos como principal forma de referirse a un correligionario político que hace parte del mismo movimiento sindical y del peronismo como movimiento político ${ }^{9}$. En el activismo de Foetra, ser compañera no solo implicaba el trabajo conjunto, sino también una colección de prácticas valoradas, expectativas y evaluaciones, que incluían dar muestras cotidianas del compromiso con la organización, demostrar participación y disponibilidad para asumir las tareas que se requirieran - asistir a actos y movilizaciones-y poner tiempo y recursos propios al servicio del sindicato, entre otros. En caso de incumplimiento, también había sanciones. La inserción como compañera permitió dilucidar analíticamente la construcción moral del activismo en Foetra a través de la definición local del compromiso que se aplicó a la antropóloga.

Entre los integrantes del pueblo mapuche, la categoría compañera también era utilizada para referirse a una relación que iba más allá de la investigación. El término weney (en mapuzungun) implicaba dar muestras de estar involucrado activamente en las demandas territoriales y por derechos, acompañar diversas tareas (elaboración de proyectos, desarrollo de talleres comunitarios, entre otros), tener disponibilidad y buena disposición frente a diversos pedidos - la mayoría de las veces no vinculados directamente con la investigación doctoral- Ser compañera abría puertas a ámbitos vedados a otros investigadores, como ceremonias, reuniones comunitarias y fiestas familiares, lo cual reforzaba la construcción moral del compromiso en términos locales.

Ser compañeras nos situó en una posición distinta a la de otros investigadores, a quienes las organizaciones consideraban externos, y nos incluyó como parte del mismo movimiento y la misma lucha, en un espacio de articulación particular gracias a nuestro compromiso. Las categorías compromiso y compañera, entendidas articuladamente, caracterizan los modos de vinculación que establecimos en nuestras investigaciones y señalan las definiciones políticas y morales del compromiso de las organizaciones. También revelan cómo moldearon nuestras prácticas de trabajo más allá de las definiciones con las que inicialmente nos acercamos a campo.

9 Sobre las estrechas vinculaciones entre sindicalismo y peronismo en Argentina, véase Torre (2012). Fernández y Carenzo (2012) también complejizan la categoría compañero a partir de su trabajo con una cooperativa de recicladores urbanos, mostrando que se trata de una categoría definida no solo en términos de su inscripción en una tradición político-ideológica determinada -el peronismo- sino que es de carácter relacional, derivada de los modos cotidianos de construcción de vínculos en la organización. 


\section{El compromiso: del posicionamiento a la práctica}

Existe en antropología una importante tradición de referentes metodológicos que -en discusión con propuestas centradas en el distanciamiento y la observación objetiva y desinteresada-, postularon como punto de partida el compromiso ético o político del investigador con los sujetos, así como el potencial de la investigación social como herramienta para el cambio social, en sus múltiples definiciones. La cuestión del compromiso como deber ético y potencial de conocimiento ha sido objeto de fuertes discusiones, como aquella entre Scheper-Hughes y D’Andrade en la década de los noventa ${ }^{10}$. Según Jimeno (2005), la vinculación entre antropología y movimientos sociales caracteriza los modos de hacer y teorizar la disciplina en América Latina ${ }^{11}$.

Sin embargo, como sostiene Hale (2006), aunque hoy el carácter situado y político del conocimiento antropológico es casi universalmente reconocido en la disciplina, los desarrollos metodológicos no están a la par de ese reconocimiento. En años recientes, según Fernández y Carenzo (2012), cobró peso un conjunto de propuestas metodológicas - especialmente en la academia estadounidense-, que tensionaron las fronteras entre investigación y activismo, así como entre "hacer” y "pensar”, y que retoman desarrollos importantes surgidos en América Latina ${ }^{12}$.

En el contexto latinoamericano, la propuesta metodológica más influyente ha sido la investigación acción participativa (IAP), más radical que la participatory action research (PAR) de Estados Unidos (Leyva Solano y Speed 2008, 38), al retomar no solo los modelos de educación popular freireana y las influencias del marxismo y su problematización del compromiso político del intelectual, sino también la idea gramsciana de praxis. La IAP entiende, en su fundamento

10 Scheper-Hughes (1995) planteó la obligación ética del antropólogo de comprometerse con los sujetos de su estudio, no solo a través de la literatura especializada o su divulgación, sino también mediante la acción política y directa en el contexto mismo de la investigación.

11 Tanto en Latinoamérica como en Argentina existen importantes discusiones en torno a la producción de conocimiento antropológico, en particular del conocimiento etnográfico y sus potencialidades. Entre otros, Visacovsky (2017), Kropff (2014), Quirós (2014), Visacovsky y Guber (2002) en Argentina, y Restrepo y Escobar (2010), Ramos (2007), Jimeno (2005) y Peirano (2004) en Latinoamérica. rrollada por Sol Tax en la Universidad de Chicago, que proponía una etnografía participativa en la que los indígenas eran tratados como coinvestigadores y el equipo de antropólogos se convertía en su grupo de estudiantes (Bennet 1996; Casas Cortés 2008). 
ideológico y epistemológico, que los miembros de las comunidades o grupos son actores sociales con voz propia, con capacidad de decisión, de reflexión y de participación en el proceso de investigación y de cambio. Los antropólogos somos herramientas que aportan a la transformación de la realidad y fortalecen las luchas de la gente con quien trabajamos; nuestro lugar es el del compromiso, entendido como el "aporte concreto de su disciplina para los fines que los movimientos populares buscan” (Fals 1981, 27).

En años recientes han cobrado relevancia planteamientos que se postulan como una continuación de los desarrollos de la IAP y de epistemologías que señalan el carácter situado del saber científico - como el pensamiento decolonial y el feminismo-. Estos apuntan a modalidades de producción de conocimiento compartido entre investigadores (académicos o de organizaciones no gubernamentales) y sujetos, aunque reconocen la existencia de "agendas" distintas entre investigadores y organizaciones, que pueden estar unidos en un compromiso conjunto pero persiguen objetivos parcialmente divergentes.

Las metodologías implicadas en las modalidades de investigación activista (Hale 2001, 2006, 2008; Speed 2006), etnografía colaborativa (Lassiter 2005, 2008; Rappaport 2007, 2008) o investigación en co-labor (Leyva 2010; Leyva y Speed 2008) proponen que todo el proceso debe ser realizado con las organizaciones o sujetos, en forma de colaboración y participación colectiva, que incluye desde la formulación del diseño del proyecto, pasando por la recolección de datos y su interpretación y análisis. En suma, donde se discutan las distinciones entre el nosotros investigadores y el ellos proveedores de datos que caracterizan muchas formas del trabajo etnográfico, especialmente en la modalidad de trabajo con informantes clave (Edelman 2009). Tales metodologías buscan hacer de esa colaboración no solo un aporte a las comunidades y organizaciones sino una "mejor etnografía”, gracias a la posibilidad de producir más y mejores datos a partir del compromiso, a la vez que análisis innovadores (Hale 2006; Rappaport 2008).

Las propuestas anteriores parten del compromiso como un posicionamiento epistémico-metodológico del investigador, sea en términos éticos/morales o políticos, con respecto a una causa de transformación social, que puede llevar al etnógrafo a definirse como militante de esa causa. Edelman (2009) propone que el compromiso (engagement o commitment) debe ser entendido como un continuo con varias dimensiones. En uno de los extremos está la investigación a la que denomina tradicional - y que otros autores caracterizan como académica-, con la que alude a la observación participante canónica, en la cual la producción de conocimiento se realiza a partir de las relaciones sociales establecidas en el trabajo de campo (Rockwell 2009), aunque los objetivos y modalidades del trabajo 
sean definidos por el investigador de manera independiente. En el otro extremo, el posicionamiento político es un punto de partida y la investigación se define y construye de manera colectiva ${ }^{13}$.

Si bien tales posturas metodológicas estaban disponibles mientras hacíamos nuestras etnografías, estas no se ajustaban plenamente a ninguna de ellas. El vínculo construido como compañeras incluía iniciativas que implicaban conexiones con las propuestas de la investigación-acción o de la etnografía colaborativa y, sobre todo, un posicionamiento político fuerte que podría pensarse como una etnografía militante o una investigación activista. Sin embargo, habíamos planteado nuestros proyectos según los tiempos, formatos y estilos requeridos por la universidad y las agencias de financiamiento que exigen la presentación de la investigación en términos académicos. Esta situación abre un espacio de discusión para problematizar dichos enfoques metodológicos y señalar las tensiones del compromiso en procesos de investigación con organizaciones sociales, considerando los modos en que los enfoques se tensionan en la práctica, en torno a tres elementos: el posicionamiento político, la definición y construcción colectiva de la investigación y el impacto esperado en la vida de las personas.

En primer lugar, tal y como propone la investigación activista, en nuestra condición de investigadoras nos situamos políticamente en relación con las demandas y las luchas de los sujetos. En la práctica, más allá de nuestra opción por el compromiso, fuimos entendiendo que este era, en cierto sentido, obligatorio para el trabajo con esas organizaciones. Sin nuestro posicionamiento en los términos señalados y sin considerarnos compañeras en sus luchas y demandas, no hubiéramos podido desarrollar nuestras indagaciones y análisis. Al mismo tiempo, a diferencia del extremo más militante de las investigaciones comprometidas en las que el académico se pone al servicio de una organización y orienta su trabajo en función de su agenda y de sus necesidades (Edelman 2009), mantuvimos cierta distancia entre nuestros objetivos investigativos y los de las organizaciones. Esta decisión se basó, en parte, en la evaluación de los obstáculos que tal extremo podría generar en el momento de validar nuestra producción de conocimiento hacia afuera y frente a los organismos de evaluación científica. También podía

13 Kropff (2014) retoma los desarrollos de Rosana Guber para señalar dos líneas fundacionales en la antropología argentina de acuerdo con la noción de compromiso que adoptan: "Una de ellas asume que el compromiso tiene que ver con la rigurosidad metodológica y teórica, mientras que la otra entiende que el compromiso implica orientar la práctica de producción de conocimiento a la transformación revolucionaria de la sociedad [...]. Según esta segunda interpretación, el antropólogo debe estar comprometido con los objetivos políticos de sus investigados" (51). En esta disyuntiva, claramente nos situamos en la segunda alternativa -aunque quizá actualizando los términos en que comprendemos el cambio social—. 
constituir un límite para el análisis de cuestiones importantes de la investigación, tales como las contradicciones de las organizaciones.

En segundo lugar, ni los objetivos ni las metas de investigación y del cambio social fueron codefinidos y codesarrollados previamente. Sin embargo, las etnografías se hicieron no solo con los sujetos, sino que también fueron pensadas para ser utilizadas por ellos. Nuestros proyectos fueron informados y negociados de maneras formales e informales con las organizaciones, por lo que no pueden ser considerados como investigaciones individuales (Fluehr-Lobban 2008). En este sentido, nuestros trabajos son respuestas académicas que tienen un compromiso político en favor de las personas y organizaciones, pero lejos de investigaciones extractivas (Leyva-Solano y Speed 2008), pretenden ser hechas con y para los sujetos, aunque no son cogenerativas. Las preguntas y problemas de investigación que nos planteamos fueron objeto de discusión en relación con las necesidades de la práctica, y no con los requisitos formales académicos que no eran parte de los requerimientos de nuestros interlocutores ni de las organizaciones. Así, por ejemplo, en la investigación de Wolanski, el vínculo con la organización estuvo basado en la demostración de los valores y prácticas que conformaban la definición local del compromiso: "poner el cuerpo" y dedicar tiempo y energía a la participación en las actividades sindicales por encima de las actividades personales. Destinar atención a la discusión de producciones académicas era considerado un lujo que reñía con las urgencias de la actividad cotidiana. Es más, en distintas ocasiones, que incluían instancias de formación sindical, el conocimiento académico o universitario era evaluado de manera abiertamente negativa, como "conocimiento por el conocimiento", y contrapuesto a la producción de conocimiento del sindicato u otras organizaciones, que se pensaba como directamente ligado a las necesidades de la práctica. Así, aunque en ambos casos discutimos nuestros interrogantes y análisis con nuestros interlocutores -y en numerosas ocasiones fueron cuestionados-, en las apretadas agendas de dirigentes y activistas la investigación colaborativa quedaba relegada frente a otras prioridades.

Finalmente, varias de estas metodologías basadas en el compromiso sostienen que las investigaciones deben tener un impacto directo y demostrable en las vidas de las personas y en determinados procesos políticos. La noción de utilidad es una vara con la que se mide constantemente nuestra práctica en las organizaciones, pero a diferencia de otros profesionales, nuestro conocimiento no suele tener una utilidad definida a priori, como es el caso de los abogados. Por este motivo, hacer evidente la importancia de nuestras investigaciones, e incluso describir nuestra actividad, resultó ser todo un desafío, que en parte saldamos mediante 
otras tareas, como compañeras, capacitadoras, colaboradoras en las prácticas propias de producción de conocimientos de Foetra y de las comunidades mapuche - muy parecidas a las de investigadores de la academia y ONG - , pero con fronteras más difusas. Al mismo tiempo, intentamos construir herramientas y formas de comunicación orientadas a hacer relevantes nuestras investigaciones en el diálogo con ellas. En el caso del comanejo, el análisis de los procesos de construcción de la diferencia cultural contribuyó a problematizar el esencialismo cristalizado en la propia definición de esta política pública y proporcionó a las comunidades herramientas para discutir y desafiar los cuestionamientos a su autenticidad, que se presentaban como el principal obstáculo para el desarrollo cotidiano de esta experiencia intercultural. En el ámbito del activismo sindical, fue más complejo hacer valer y legitimar la contribución académica de la antropología en un contexto en el que - a diferencia de los temas étnicos y de pueblos originarios- no siempre se reconoce su competencia. No obstante, el foco en las relaciones intergeneracionales permitió intervenir en espacios de formación y activismo, así como poner en cuestión las divisiones entre jóvenes y viejos y distintas acusaciones de los mayores hacia los jóvenes.

Entendemos que nuestras investigaciones se dieron en un marco particular que nos permitió contribuir de diversas maneras con las organizaciones - pericias judiciales, capacitaciones, talleres, producciones didácticas, etc.pero no fueron el eje principal de articulación y trabajo conjunto. En este proceso, el compromiso, lejos de ser una metodología de investigación preestablecida, se fue redefiniendo en la práctica a partir de los vínculos, negociaciones e interacciones con nuestros interlocutores.

\section{Incomodidades y potencialidades del compromiso en la producción de conocimiento con los sujetos y colectivos}

Al posicionarnos desde el compromiso, buscamos mantener esa difícil independencia analítica que pregonan los propulsores de la investigación activista. No produjimos tesis que las organizaciones hubieran escrito sobre sí mismas, intentando conscientemente evitar un trabajo que fuera una oda o, en los términos de Edelman (2009), que tuviera un enfoque cosmético (249), es decir, que reprodujera las narrativas y representaciones que las organizaciones construyen en 
su práctica. Nuestro posicionamiento podía implicar que nos convirtiéramos en una suerte de intelectuales orgánicas de estos colectivos, al plasmar en nuestras etnografías la visión que proyectan sobre sí mismos e invisibilizar contradicciones internas. Ese fue uno de los principales problemas que enfrentamos, pero también fue parte del aporte de nuestras investigaciones. En numerosas ocasiones nuestras miradas como antropólogas no coincidieron con las visiones $\mathrm{u}$ opiniones de las organizaciones con respecto a ciertos procesos; sin embargo, decidimos mantenerlas, lo que exigió un difícil equilibrio entre la producción de análisis críticos sobre las prácticas y los procesos que etnografiamos, así como el respeto por los códigos y las trastiendas definidas localmente, y el objetivo de aportar desde esa crítica a las propias organizaciones. Hacer de estas tensiones un objeto de indagación y reflexión, como propone Hale (2001), también mostró su productividad en la generación de conocimiento.

Al hacer etnografía con organizaciones, una primera tensión que es necesario sortear es la regulación que estas ejercen sobre la producción de conocimiento acerca de sí mismas. Esto fue particularmente notorio en el caso del activismo sindical. Los vínculos personales de confianza y amistad establecidos previamente facilitaron el acceso a ciertas instancias públicas o semipúblicas de la vida sindical, donde se realizó un número de entrevistas (en general a dirigentes o activistas especialmente designados como portavoces), y en ocasiones participar de algún acto o espacio de formación organizado por el sindicato. Se establecían así modos tolerados y controlados de investigación por parte de externos, que limitaban los espacios y tiempos accesibles a una observación participante en el sentido canónico. El primer paso para conocer la trastienda oculta y vedada a la mirada pública fue la incorporación como colaboradora docente en los cursos de formación sindical de la Secretaría de Cultura y Capacitación, a partir de lo cual le fue posible tomar parte en debates con un gran número de activistas del sindicato y comenzar a discernir la coexistencia de distintos posicionamientos políticos, las negociaciones implicadas en la organización de cada actividad sindical, las diferencias internas y la manera como eran saldadas. Aunque la organización ejercía un efectivo control sobre los espacios y relaciones que hacía visibles a los investigadores, trascender esa frontera fue posible mediante el compromiso.

En el caso del comanejo, el análisis de la construcción de la diferencia cultural a partir del acceso a ciertas prácticas y procesos comunitarios vedados a los de afuera fue central para mostrar lo lento, conflictivo y complejo de la construcción de lazos comunitarios que habían sido trastocados por políticas estatales de negación de la existencia indígena en la región y por fuertes procesos 
de invisibilización y estigmatización, en relación con los requisitos burocráticos del Estado para su reconocimiento, como la obtención de la personería jurídica. La participación en la trastienda reveló cómo lo formal y burocrático se da con anterioridad al proceso de fortalecimiento comunitario, lo que lleva a que desde afuera sea visto como forzado y oportunista.

Este modo de vinculación supuso una segunda tensión para nuestras investigaciones, en relación con la escritura y la publicación. Acceder a la trastienda permitió conocer conflictos, tensiones internas, divisiones y desacuerdos, así como prácticas que eran condenadas públicamente, pero que en ciertos contextos eran usuales y toleradas. En algunas perspectivas antropológicas, el conflicto y las contradicciones son precisamente el material buscado para la reflexión, desde el supuesto de que los sujetos no son del todo conscientes de estos, y de que el investigador, desde la externalidad, es quien puede señalarlos. Esta es, quizá, parte de la lógica académica que contrasta más abiertamente con los objetivos de las organizaciones ya que, como señalan Leyva y Speed $(2008,47)$, los conflictos dentro de la organización son un capital social muy importante para estas, para sus estrategias y tácticas de lucha, y son aspectos que deben ser tratados en el lugar y momento oportunos con las personas adecuadas. Asumir una postura comprometida supuso silenciar datos que pudieran implicar consecuencias negativas para los sujetos y organizaciones con los que trabajamos, como reconstruye Fernández (2010) en el caso de su investigación con una empresa recuperada de la ciudad de Buenos Aires. A pesar de esas limitaciones, al adoptar una postura comprometida y de trabajo conjunto logramos conocer más de las realidades internas de las organizaciones que si hubiéramos mantenido una posición de externalidad, hecho que fue central en el momento de producir conocimiento etnográfico.

En el caso del comanejo, cobró particular importancia una tercera tensión relacionada con la publicación y el manejo de los datos. Desde el comienzo del trabajo de campo fue necesario actuar con cuidado porque se trataba de un tema sensible, que involucraba comunidades indígenas en un área protegida que históricamente había sido considerada "sin indios", y en la que estaban empezando a surgir reclamos territoriales e identitarios. Fernández (2010) define estos casos como procesos políticos calientes que se van dando mientras hacemos nuestras investigaciones y que nos permiten ser testigos de los avances, retrocesos, quiebres, disputas, festejos, conflictos, acuerdos, obstáculos y cambios de una organización social, pero que también nos ponen ante el desafío de lidiar con los cambios de posición y de opinión de los sujetos. Esto tiene implicaciones directas en la producción de conocimiento etnográfico, que suele quedar obsoleto 
rápidamente por las dinámicas particulares y cambiantes de estos procesos, y nos demanda reflexionar acerca de qué decir o no para no obstaculizarlos. Así, durante la investigación sobre el comanejo, fueron conformándose nuevas comunidades indígenas, y cada uno de estos procesos generó conflictos particulares con la administración del parque. En este contexto, era común que el contenido de una publicación quedara desfasado hasta en la cantidad de comunidades que conformaban el comanejo, o que hiciera alusión a fuertes tensiones y conflictos que meses después eran olvidados por los sujetos, pero que habían requerido de un posicionamiento claro de la investigadora. Ejemplos como este nos hacen testigos de los conflictos cotidianos entre los involucrados, quienes están aprendiendo a conducir estas experiencias sobre la marcha. Asimismo, estos procesos políticos calientes suponen el desafío de situarnos en el marco de conflictos particulares y evaluar la utilidad de nuestras investigaciones en su resolución.

La cuarta tensión que identificamos es la implicación afectiva que se va dando a lo largo del proceso etnográfico y que muchas veces termina opacando, en la práctica, el objetivo principal con el que llegamos a las organizaciones. El que nos reconocieran como compañeras facilitó acceder a espacios privilegiados, pero también implicó que nuestro lugar como investigadoras quedara desdibujado en la identidad de compañeras que ayudaban y acompañaban los procesos de organización y fortalecimiento de estos colectivos. Fernández (2010) sostiene que el trabajo etnográfico se basa en el encuentro, en el establecimiento de relaciones personales sostenidas en el tiempo, en la posibilidad que abre este conocimiento. Explica que, cuando trabajamos con organizaciones sociales, estas relaciones personales están atravesadas por vínculos políticos y, en consecuencia, nos involucran política, ética y afectivamente, y nos exigen tomar posición en situaciones de tensión y negociación, e incluso confrontación y padecimiento, por lo que nuestra presencia supone siempre una mirada implicada ${ }^{14}$. En concordancia con esta autora, nos interesa pensar cómo, muchas veces, nuestro trabajo etnográfico quedó invisibilizado en lo cotidiano. En tanto el vínculo nos permitía circular por espacios de cierto privilegio, al mismo tiempo suponía que muchas veces nuestros interlocutores olvidaran que uno de nuestros objetivos en esos espacios era la realización de una investigación. En los hechos, esto implicaba trasladar

14 En este mismo sentido, en otro artículo la autora distingue dos aspectos en el extrañamiento que suele exigirse a la antropología como garantía de una "buena" investigación: una distancia ontológica entre nosotros (investigadores) y ellos (sujetos de investigación), que establece distancia (afectiva, política, profesional) y que se superpone a una distancia "metodológica", que permite la reflexión crítica (Fernández y Carenzo 2012, 30). Siguiendo a estos autores, entendemos que devenir compañeras fue un modo de poner en discusión la primera dimensión de esa distancia, sin por eso perder la segunda, referida a la posibilidad de análisis y crítica de las prácticas y los procesos sociales. 
hacia nosotras mucha de la vigilancia y el cuidado sobre aquello que escribíamos o hacíamos público.

Para finalizar, nos interesa abordar una última tensión que identificamos en ambas investigaciones, vinculada al género de las investigadoras. Aunque no hacemos una reflexión específica sobre las relaciones de género en nuestras tesis, estas sin duda han incidido en los procesos de nuestras investigaciones, en nuestros trabajos de campo y en la construcción del compromiso. Tanto el ámbito sindical como el rural son predominantemente masculinos y en ambos existen roles y expectativas diferenciales asignados a varones y mujeres, así como espacios en los cuales es más o menos difícil circular como mujer. En la investigación sobre el activismo sindical, se hizo patente el hecho de que, aunque en el ámbito del activismo los dos géneros se hallaban representados más o menos equitativamente, las dirigencias gremiales fueran espacios preponderantemente masculinos. Este hecho contribuyó a mantener la opacidad de las instancias dirigenciales, de modo que la diferencia de género — así como la generacional-, reforzaba los mecanismos establecidos para excluir a la investigadora de ellas. Para Trentini, por su parte, transitar en las comunidades indígenas como investigadora puso en tensión regulaciones locales sobre las actividades y espacios masculinos y femeninos, al ser una mujer en situaciones localmente definidas como masculinas.

A pesar de las dificultades, estas tensiones del compromiso fueron fundamentales en la producción de miradas analíticas renovadas, que transformaron nuestras preguntas y puntos de partida, cuestionando algunos de los supuestos (políticos, teóricos y metodológicos) con los que habíamos arribado a campo, y señalando líneas de análisis novedosas.

Así, en el caso de la etnografía sobre el comanejo, la forma de relacionarnos con las comunidades indígenas resultó un desafío en el momento de producir conocimiento, en tanto la construcción de lazos que facilitaban un importante nivel de confianza complicó al principio la problematización sobre ciertos aspectos. En retrospectiva, podríamos considerar que la vinculación afectiva y política de la etnógrafa generó al comienzo de la investigación una mirada dicotómica entre dos sectores aparentemente homogéneos y opuestos: comunidades indígenas frente a la Administración de Parques Nacionales, partiendo del supuesto de que la implementación de un comanejo perpetuaba la hegemonía institucional y no daba lugar a una verdadera participación de las comunidades en la toma de decisiones. En otras palabras, existía una mirada idealizada de las comunidades y cierta demonización de la institución. Sin embargo, la posibilidad de participar de diversas instancias cotidianas - tanto institucionales como comunitarias- llenas de 
contradicciones, disputas, avances y retrocesos permitió complejizar esta mirada inicial. Fue posible observar las divergencias dentro de la propia administración del parque, así como entre los miembros de las comunidades y organizaciones mapuche, en su forma de entender y construir cotidianamente el comanejo. Esas instancias ayudaron a poner en cuestión las propias categorías de comanejo y de comunidad indígena, mostrando las relaciones y los múltiples intereses que se iban poniendo en juego, y así entender el comanejo como una arena de disputa política que permitía reconfigurar los límites y posibilidades de la acción política de las comunidades.

Por su parte, en el caso de la etnografía sobre el sindicato, el modo de vinculación establecido llevó a redefinir el objeto de la investigación. En un principio, el proyecto de investigación postulaba la intención de indagar en las prácticas políticas de los trabajadores telefónicos, imaginando desde lecturas previas la centralidad de los espacios de trabajo en la construcción de la política sindical cotidiana. La vinculación con los espacios de activismo hizo visibles categorías de activismo sindical que no estaban previstas y el lugar que ocupaban los activistas - diferenciados del conjunto de los trabajadores-en la construcción cotidiana del sindicato. La multiplicidad de cuadros intermedios y de distintos modos de tomar parte del activismo sindical, así como las carreras militantes de los jóvenes activistas y las relaciones entre distintas generaciones, no habían sido el foco de otros trabajos etnográficos de investigación. La propia necesidad de aprender a mostrar el compromiso como antropóloga contribuyó a iluminar los valores y actitudes que los activistas del sindicato ponían en acto en su actividad cotidiana, evaluando y legitimando los accionares propios y ajenos y plasmando en esos juicios orientaciones políticas y construcciones morales. Encarnar el compromiso en la práctica investigativa y en los distintos espacios nutrió el análisis de esa construcción moral de la lucha sindical.

\section{A modo de conclusión}

En este artículo reflexionamos sobre los modos de relación que establecimos con dos organizaciones sociales en el marco de nuestras investigaciones doctorales, y cómo esos modos de relación se vincularon con la producción de conocimiento etnográfico. Propusimos desplazar la mirada desde el compromiso como postura ética/académica a la construcción conjunta de la investigación comprometida, a partir de intereses, definiciones y sentidos, tanto de las etnógrafas como de los 
sujetos y organizaciones, iluminando las incomodidades y tensiones de ese proceso y a la vez postulando sus potencialidades en la producción de conocimiento.

Encontramos que no existían recetas metodológicas predefinidas, sino tensiones, recaudos y desafíos comunes que requerían ponerse en diálogo con las expectativas y propuestas de los sujetos y organizaciones, así como con sus propias definiciones del compromiso. Es importante señalar que las modalidades de investigación y el compromiso construidos tampoco adquirieron formas definitivas. Por el contrario, esta reflexión nos permite también situar la temporalidad de los acuerdos y compromisos gestados y sus redefiniciones.

En efecto, una vez terminado el objetivo concreto de hacer una investigación, nos encontramos que el compromiso construido no se podía concluir de un día para el otro, no solo por la multiplicidad de espacios cocreados y transitados en conjunto, sino por las implicaciones afectivas y políticas en las cuales asumimos objetivos propios, y entablamos relaciones personales; así como también por las expectativas de continuidad de los sujetos y las organizaciones. El siguiente periodo es de negociación y reposicionamiento. Implica redefinir los compromisos creados, a la vez que precisar nuevas agendas de investigación. Aceptamos y aprendimos que esos compromisos construidos suponen un límite para futuras investigaciones, por cuanto definen rumbos posibles y otros conflictivos, por ejemplo, con organizaciones opuestas en el espectro político, que pueden ser vistos con recelo e incluso como formas de traición a los vínculos previos, si no son explicados y negociados con anterioridad. También comenzamos a renegociar nuestra presencia en las organizaciones, a elegir otros modos de estar presentes, menos en la vida cotidiana que supone la etnografía, pero manteniendo las tareas de extensión y transferencia, así como el acompañamiento en movilizaciones y acciones de protesta y reivindicación. En paralelo, la modificación del contexto político del país en los últimos años también influyó en las necesidades y definiciones de las organizaciones - tanto en el caso del sindicato como de las comunidades mapuche-, y ellas fueron rediseñando sus objetivos, demandas y estrategias y, en el proceso, resituando lo que se esperaba de nuestro compromiso.

Finalmente, el vínculo generado hace que nuestros títulos de doctorado tengan un peso estratégico, en términos del reconocimiento de nuestra palabra en el momento de acompañar algunos procesos actuales de las organizaciones. Esta nueva situación, aún incierta, nos llevó a reflexionar sobre el compromiso en este artículo, cuando se nos vuelve a redefinir en el mismo espacio que venimos transitando hace más de diez años y se nos presenta de nuevo el desafío de pensar la producción de conocimiento en relación con las organizaciones que nos interpelan, nos demandan y nos involucran directamente en sus disputas 
cotidianas por derechos y reivindicaciones. Reflexionar sobre el camino transitado y, sobre todo, volver a las incomodidades y desafíos y las resoluciones que les dimos devienen centrales en el proceso de seguir aportando a la producción de conocimiento crítico y comprometido.

\section{Agradecimientos}

Agradecemos especialmente a María Inés Fernández Álvarez y a los integrantes del equipo editorial de la Revista Colombiana de Antropología, por la lectura atenta, crítica y comprometida de versiones previas de este artículo, que sin duda han sido una enorme contribución a él.

\section{Referencias}

Bennet, John. 1996. "Applied and Action Anthropology. Ideological and Conceptual Aspects”. Current Anthropology 36: 23-39.

Cañumil, Pablo y Ana Ramos. 2011. "Knowledge Transmission through the Renü”. Collaborative Anthropologies 4 (1): 67-89.

Casas Cortés, María Isabel. 2008. "Etnografías made in USA: rastreando metodologías disidentes". En Miradas, encuentros y críticas antropológicas, coordinado por Aitzpea Leizaola y Jone Miren Hernández, 165-171. https://www.ankulegi.org/wp-content/ uploads/2012/03/130302Casas-Cortes.pdf.

Edelman, Marc. 2009. "Synergies and Tensions between Rural Social Movements and Professional Researchers”. Journal of Peasant Studies 36 (1): 245-265.

Etchemendy, Sebastián y Ruth Berins Collier. 2008. "Golpeados pero de pie. Resurgimiento sindical y neocorporativismo segmentado en Argentina (2003-2007)”. Revista Post-Data 13: 145-192.

Fals Borda, Orlando. 1981. "La ciencia y el pueblo”. En Investigación participativa y praxis rural, editado por Francisco Vio Grossi, Vera Gianotten y Ton de Wit, 279-287. Lima: Mosca Azul.

Fernández Álvarez, María Inés. 2010. “Desafíos de la investigación etnográfica sobre procesos políticos ‘calientes”. (Con)textos Revista d’Antropología 4: 80-89.

—. 2016. Hacer juntos. Dinámicas, contornos y relieves de la política colectiva. Buenos Aires: Biblos. 
Fernández Álvarez, María Inés y Sebastián Carenzo. 2012. “Ellos son los compañeros del Conicet’: el vínculo con organizaciones sociales como desafío etnográfico”. Publicar en Antropología y Ciencias Sociales X (XII): 9-33.

Fluehr-Lobban, Caroline. 2008. "Collaborative Anthropology as Twenty-First-Century Ethical Anthropology”. Collaborative Anthropologies 1: 175-182.

García, Analía y Sebastián Valverde. 2007. "Políticas estatales y procesos de etnogénesis en el caso de poblaciones mapuche de Villa La Angostura, provincia de Neuquén, Argentina”. Cuadernos de Antropología Social 25: 111-132.

Hale, Charles. 2001. “What is Activist Research?”. Social Science Research Council 2 (1-2): 13-15.

-. 2006. "Activist Research vs. Cultural Critique: Indigenous Land Rights and the Contradictions of Politically Engaged Anthropology”. Cultural Anthropology 21: 96-120.

-. ed. 2008. Engaging Contradictions. Theory, Politics and Methods of Activist Scholarship. Los Ángeles: University of California Press.

Jimeno, Myriam. 2005. "La vocación crítica de la antropología en Latinoamérica”. Antípoda 1: $43-65$.

Kropff, Laura. 2014. "Acerca del posicionamiento: investigación activista, crítica cultural o activismo crítico". En Antropologías contemporáneas. Saberes, ejercicios y reflexiones, editado por Débora Betrisey y Silvina Merenson, 51-66. Buenos Aires, Madrid: Miño y Dávila.

Lassiter, Luke Eric. 2005. “Collaborative Ethnography and Public Anthropology”. Current Anthropology 46 (1): 83-106.

-. 2008. "Moving Past Public Anthropology and Doing Collaborative Research". NAPA Bulletin 29: 70-86.

Leyva Solano, Xochitl. 2010. “¿Academia versus activismo? Repensarnos desde y para la práctica teórico-política”. En Conocimientos y prácticas políticas: reflexiones desde nuestras prácticas de conocimiento situado, editado por Xochitl Leyva Solano et al., 1-29. Chiapas, Ciudad de México, Lima y Ciudad de Guatemala: Ciesas, PDTG-USM, UNICACH. http:// www.ceapedi.com.ar/imagenes/biblioteca/libros/319.pdf.

Leyva Solano, Xochitl y Shannon Speed. 2008. "Hacia la investigación descolonizada: nuestra experiencia de co-labor". En Gobernar (en) la diversidad: experiencias indígenas desde América Latina. Hacia la investigación de colabor, coordinado por Xochitl Leyva, Araceli Burguete y Shannon Speed, 34-59. Ciudad de México, Quito, Ciudad de Guatemala: Ciesas, Flacso Ecuador y Flacso Guatemala.

Méndez, Laura. 2005. "Mi pasado me condena. Chilenos e indígenas en el Nahuel Huapi 18801935”. Ponencia presentada en el VI Congreso de Historia Política y Social de la Patagonia Argentino-Chilena, Universidad Nacional de la Patagonia San Juan Bosco, Trelew, 13-15 de octubre.

Peirano, Mariza. 2004. “A favor de la etnografía”. En La antropología brasileña contemporánea. Contribuciones para un diálogo latinoamericano, compilado por Alejandro Grimson, Gustavo Lins Ribeiro y Pablo Semán, 323-356. Buenos Aires: Prometeo.

Quirós, Julieta. 2014. “Etnografiar mundos vividos. Desafíos de trabajo de campo, escritura y enseñanza en antropología”. Publicar 17: 47-65. 
Radovich, Juan Carlos y Alejandro Balazote. 2009. "El pueblo mapuche contra la discriminación y el etnocidio". En Historia de las familias Paichil Antriao y Quintriqueo de la costa norte del lago Nahuel Huapi, compilado por Gerardo Ghioldi, 35-51. Villa La Angostura: Archivos del Sur, Biblioteca Popular Osvaldo Bayer.

Ramos, Alcida Rita. 2007. “¿Hay lugar aún para el trabajo de campo etnográfico?”. Revista Colombiana de Antropología 43: 231-261.

Rappaport, Joanne. 2008. "Beyond Participant Observation: Collaborative Ethnography as Theoretical Innovation”. Collaborative Ethnographies 1: 1-31.

—. 2007. “Más allá de la escritura: la epistemología de la etnografía en colaboración”. Revista Colombiana de Antropología 43: 197-229.

Restrepo, Eduardo y Arturo Escobar. 2010. "Red de Antropologías del Mundo: intervenciones en imaginación teórica y política de la práctica política”. En Conocimientos y prácticas políticas: reflexiones desde nuestras prácticas de conocimiento situado, editado por Xochitl Leyva Solano et al., 133-165. Chiapas, Ciudad de México, Ciudad de Guatemala, Lima: Ciesas, PDTG-USM, UNICACH.

Rockwell, Elsie. 2009. La experiencia etnográfica: historia y cultura en los procesos educativos. Buenos Aires: Paidós.

Rodríguez, Mariela. 2010. De la "extinción" a la autoafirmación: procesos de visibilización de la Comunidad Tehuelche Camusu Aike (provincia de Santa Cruz, Argentina). Washington DC: ProQuest-Georgetown University.

Scheper-Hughes, Nancy. 1995. "The Primacy of the Ethical: Propositions for a Militant Anthropology”. Current Anthropology 36 (3): 409-440.

Senén González, Cecilia y Julieta Haidar. 2009. "Los debates acerca de la 'revitalización sindical' y su aplicación en el análisis sectorial en Argentina”. Revista Latinoamericana de Estudios del Trabajo (2. da época) 22: 5-31.

Speed, Shanon. 2006. "Entre la antropología y los derechos humanos. Hacia una investigación activista y comprometida críticamente”. Alteridades 16 (31): 73-85.

Torre, Juan Carlos. 2012. Ensayos sobre sindicalismo y peronismo. Buenos Aires: Siglo XXI.

Trentini, Florencia. 2016. "Procesos de construcción de la diferencia cultural en el comanejo del Parque Nacional Nahuel Huapi”. Revista de Estudios Sociales 55: 32-44.

—. 2014. "Pueblos indígenas y áreas protegidas: procesos de construcción de identidades y territorialidades en el comanejo del Parque Nacional Nahuel Huapi”. Tesis de Doctorado de la Facultad de Filosofía y Letras de la Universidad de Buenos Aires, orientación Antropología Sociocultural.

Visacovsky, Sergio E. 2017. "Etnografía y antropología en Argentina: propuestas para la reconstrucción de un programa de investigación de lo universal”. Antípoda 27: 65-91.

Visacovsky, Sergio E. y Rosana Guber, comps. 2002. Historias y estilos de trabajo de campo en la Argentina. Buenos Aires: Antropofagia. 
Wolanski, Sandra. 2017. "Un sindicato que lucha. Narrativas del pasado y construcciones morales entre los activistas del sindicato de las telecomunicaciones de Buenos Aires”. Revista Latinoamericana de Antropología del Trabajo 1 (2).

-. 2015. "Construir el sindicato. Trabajo militante y generaciones activistas en el sindicato telefónico de Buenos Aires”. Tesis de Doctorado de la Facultad de Filosofía y Letras de la Universidad de Buenos Aires, orientación Antropología Sociocultural. 